\title{
Alimentación de cuyes en la fase de crecimiento en base a gramíneas tropicales de Morona Santiago
}

\section{Feeding of guinea pigs in the growth phase based on tropical grasses from Morona Santiago}

Luis Rojas Oviedo. ${ }^{1}$, Tamia Noboa Abdo. ${ }^{2}$, Segundo Shagñay Rea. ${ }^{3}$, \& Luis Condo Plaza. $^{4}$

\begin{abstract}
DOI: https://doi.org/10.33262/concienciadigital.v3i2.2.1245
The feeding of guinea pigs in the growth phase based on tropical grasses was developed on the ESPOCH farms Sede Morona Santiago, for which 20 guinea pigs 60 days old were used, which were distributed in five treatments (Tripsacum laxum, Pennisetum sp, Pennisetum purpureum and Axonopus scoparius) including the control and four repetitions, each experimental unit was made up of one animal, which had an average weight of $409.6 \mathrm{~g}$. to which they were randomly housed in an individual metabolic cage to observe the productive behavior and use of the pasture, and to assign the treatments at random to each guinea pig. Dehydrated feed was supplied daily after recording its weight, in the same way forage residues and excreta were weighed, data that were used for the analysis. Once the information obtained was compiled and processed, it was possible to notice that the weight of the guinea pigs after 60 days of investigation, consumption of dry matter, quantity of feces produced, and dry matter used did not register significant differences, but the use of protein, fat, fiber, Acid Detergent Fiber (FDA) and neutral detergent fiber (FDN) registered statistical differences. Concluding that the balance has a greater amount of protein and fat used, although the fiber content is scarce, in the same way the availability of FDN and FDA in the Tripsacum laxum grass is in a lower proportion when contrasting with the other grasses used in the investigation.
\end{abstract}

Key words: Cavia porcellus, Tropical grasses, Food, Protein, Fiber.

\footnotetext{
${ }^{1}$ Escuela Superior Politécnica de Chimborazo, Macas Ecuador, luis_ro26@hotmail.com

${ }^{2}$ Escuela Superior Politécnica de Chimborazo, Macas Ecuador, taminobo31@gmail.com,

${ }^{3}$ Escuela Superior Politécnica de Chimborazo, Macas Ecuador,luis_ro26@hotmail.com

${ }^{4}$ Universidad Regional Amazónica IKIAM, Tena Ecuador, luis.condo@ikiam.edu.ec
} 


\section{Resumen.}

La alimentación de cuyes en la fase de crecimiento en base a gramíneas tropicales se desarrolló en los predios de la ESPOCH Sede Morona Santiago, para lo cual se utilizaron 20 cuyes de 60 días de edad, los mismos que se distribuyeron en cinco tratamientos (Tripsacum laxum, Pennisetum sp, Pennisetum purpureum $y$ Axonopus scoparius) incluido el control y cuatro repeticiones, cada unidad experimental estuvo conformado por un animal el mismo que tuvo un peso promedio de $409.6 \mathrm{~g}$. a los cuales se le alojaron aleatoriamente en una jaula metabólica individual para observar el comportamiento productivo y aprovechamiento del pasto, y asignar los tratamientos al azar a cada cobayo. Diariamente se suministró alimento deshidratado previo el registro de su peso, de la misma manera se pesó los residuos de forraje y las excretas, datos que sirvieron para el análisis. Una vez recopilada y procesada la información obtenida se pudo notar que el peso de los cuyes a los 60 días de investigación, consumo de materia seca, cantidad de heces producida, materia seca aprovechada no registraron diferencias significativas no así, el aprovechamiento de la proteína, grasa, fibra, Fibra Detergente Ácida (FDA) y fibra detergente neutra (FDN) registró diferencias estadísticas. Concluyéndose que el balanceado dispone de mayor cantidad de proteína y grasa aprovechada, aunque el contenido de fibra es escaso, de la misma manera la disponibilidad de FDN y FDA en el pasto Tripsacum laxum está en menor proporción al contrastar con el resto de pastos utilizados en la investigación.

Palabras claves: Cavia porcellus, Pastos tropicales, Alimentación, Proteína, Fibra.

\section{Introducción.}

El Cavia porcellus, es un mamífero roedor nativo de América del Sur; distintas tribus aborígenes manejaron esta especie hace más de 500 años como mascota; desciende de la especie salvaje Cavis cutlerí. La cultura Paracas en el período de las cavernas por los años 250 a 300 a.c, se alimentaba de carne de este roedor (Coronado, 2007).

El cuy es dócil, fácil de manejar; herbívoro y monogástrico; tiene un estómago donde inicia su digestión enzimática y un ciego funcional en donde se produce una fermentación bacteriana de forrajes y granos. En la actualidad tiene múltiples usos además de ser mascota y animal experimental. (Chauca, 1997 y Argote et al., 2007).

Su capacidad de adaptación permite encontrarse desde el nivel del mar hasta los 4,500 metros sobre el nivel del mar. (Solari, 2010). 
El cuy es herbívoro, su alimentación se basa en forraje verde y el suministro de diferentes tipos de alimento, muestra preferencia por el forraje (Zaldívar y Rojas, 1968); la alimentación influye en la producción y representa del $70 \%$ al $80 \%$ del coste de producción (Solari, 2010).

Los requerimientos nutricionales del cuy, permite formular raciones balanceadas que satisfagan las necesidades de mantenimiento, crecimiento y producción e cada una de las fases fisiológicas (Chauca, 1997).

En la provincia Morona Santiago se evidencia la crianza del cuy inti, siendo una alternativa para mejorar sus ingresos económicos. Su alimentación se basa en forrajes de la zona como: el Gramalote, Guatemala, Elefante y Maralfalfa, siendo estas gramíneas de mayor frecuencia.

\section{Materiales y Métodos}

La alimentación de los cuyes a partir de los 60 días de edad se realizó a base de Tripsacum laxum (T1), Pennisetum sp (T2), Pennisetum purpureum (T3) y Axonopus scoparius (T4) frente a un control (balanceado); se desarrolló en los predios de la ESPOCH Sede Morona Santiago a 1150 m.s.n.m, con una temperatura ambiental de $24.6{ }^{\circ} \mathrm{C}$ y una humedad relativa de $260 \mathrm{~mm}$ anual; para lo cual se utilizaron 20 animales distribuidos en 5 tratamientos incluido el control control con 4 repeticiones, los cuales fueron ubicados en jaulas individuales durante 60 días. Las unidades experimentales se distribuyeron al azar, razón por lo que los resultados experimentales se analizaron bajo un Diseño Completamente al Azar cuyo modelo lineal aditivo fue: $Y i j=u+T i j+$ Eij.

Dónde: Yij: Valor estimado de la variable, u: Media general, Ti: efecto de los diferentes tipos de gramíneas tropicales, y Eij: Efecto de la aleatorización de las unidades experimentales en el campo experimental (error experimental), separación de medias según Tukey, modelo que se corrió con el software INFOSTAT Estudiantil.

\section{Resultados y Discusión}

Al inicio de la investigación los cuyes que se sometieron al balanceado, Tripsacum laxum, Pennisetum sp, Pernisetum purpureum y Axonopus scoparius pesaron 425.00, 385.75, $392.63,416.38$ y $428.38 \mathrm{~g}$ en promedio respectivamente, valores que demuestran homogeneidad, siendo adecuado el proceso de investigación. Los cuyes al destete deben pesar entre 159 y 259 g (Xicohtencatl-Sánchez, 2013), valor inferior al registrado en el presente estudio.

Luego de los 60 días de investigación en la fase de crecimiento, los cuyes que recibieron balanceado, Tripsacum laxum, Pennisetum sp, Pernisetum purpureum y Axonopus scoparius tuvieron un peso vivo en promedio de 724.25, 625.75, 695.25, 584.50 y 713.88 
g respectivamente, valores entre los cuales no difieren significativamente $(\mathrm{P}>0.05)$, aunque se puede apreciar que el mayor peso que alcanzaron los cuyes fue al utilizar el balanceado seguido del Axonopus scoparius, esto puede deberse a la digestibilidad de la materia seca y nutrientes. Los cuyes en ayunas alcanzaron un peso de $980 \mathrm{~g}$ (XicohtencatlSánchez, 2013), siendo superiores al encontrado en el presente trabajo, esto se debe a que los cuyes tienen una edad de 120 días. El peso de los cuyes al utilizar hoja de maíz a los 90 días fue de $810.50 \mathrm{~g}$ (Bonilla, S 2014), Al emplear el forraje verde hidropónico de maíz en la etapa de crecimiento de igual manera registró 810 g (Gómez, M. 2007); la utilización de forraje verde hidropónico de maíz registró $992 \mathrm{~g}$ de peso vivo (Casa, C. 2008); al emplear las hojas de maíz registraron pesos finales de 826,9 g (Sánchez, A. et al. 2009); al utilizar las hojas de dos variedades de maíz en la alimentación de los cuyes hasta los 120 días de edad, alcanzó pesos entre 0,96 y 1,19 kg (Sayay, M. 2010) y al emplear ensilaje de maíz en la alimentación de los cobayos determinó pesos de $919 \mathrm{~g}$ (Sandoval, H. 2013), pudiendo señalar que existe variación debiéndose prácticamente a la variación genética y el manejo de cada investigación.

El consumo de balanceado, Tripsacum laxum, Pennisetum sp, Pernisetum purpureum y Axonopus scoparius en los cuyes en la fase de crecimiento fue de 2230.63, 2079.91, 2266.68, 2296.76 y 2274.11 g durante 60 días, valores que entre si no difieren significativamente $(\mathrm{P}>0.05)$ entre las gramíneas forrajeras, pudiéndose notar mayor consumo en el Pennisetum purpureum y el menor consumo en el Trisacum laxum, esto posiblemente se debe a la palatabilidad de cada uno de los pastos. En la etapa de crecimiento el consumo de materia seca de los cuyes fue de $0.93 \mathrm{~kg}$ (Londo. V., Usca, J. Díaz, H. y Trujillo, V. 2014), al utilizar las zeolitas durante 75 días, siendo inferior al registrado en el presente estudio.

Esto quizá se deba a la calidad y palatabilidad de los alimentos; de la misma manera se asevera que el consumo de materia seca fue de $3174 \mathrm{~g}$ (Acosta, A. V. Díaz, H. y Trujillo, V. 2010) superior al alcanzado en esta investigación, posiblemente se deba al grupo genético de los cuyes. Aunque también se puede manifestar que el consumo de alimento en base seca fue de $2300 \mathrm{~g}$, siendo similar al registrado en el presente trabajo investigativo (Villa, S. Díaz, H. Usca, J. (2014).

De la misma manera se corrobora que el consumo de materia seca en cuyes es de $2160 \mathrm{~g}$ (Bonilla, S. Usca, J. Díaz, H. 2014).

La producción de excretas de los cuyes durante 60 días en la fase de crecimiento, al aplicar balanceado, Tripsacum laxum, Pennisetum sp, Pernisetum purpureum y Axonopus scoparius fue de $345.11,365.29,362.19,323.28$ y $338.20 \mathrm{~g}$, valores entre los cuales no existen diferencias relevantes $(\mathrm{P}>0.05)$ entre las especies forrajeras, notándose que la mayor producción de excretas fue al aplicar Trisacum laxum y el menor cantidad, al aplicar Pennisetum purpureum, esto quizá se deba a que este pasto tenga mayor digestibilidad a la cosecha. 
Al utilizar balanceado y los forrajes como: Tripsacum laxum, Pennisetum sp, Pernisetum purpureum y Axonopus scoparius se retuvo en los cuyes 1885.52, 1714.62, 1904.49, 1973.47 y $1935.90 \mathrm{~g}$ de materia seca durante los 60 días en la fase de crecimiento, valores entre los cuales no hay diferencias relevantes $(\mathrm{P}>0.05)$ entre los diferentes alimentos, determinándose el mayor aprovechamiento de la materia seca al utilizar Pennisetum purpureum y el menor aprovechamiento al aplicar Trisacum laxum, esto posiblemente se deba a la digestibilidad que tienen los pastos en el organismo de los monogástricos.

La cantidad de proteína aprovechada en los cuyes en la fase de crecimiento durante los 60 días al utilizar balanceado, Tripsacum laxum, Pennisetum sp, Pernisetum purpureum y Axonopus scoparius fue de 305.45, 133.40, 151.17, 149.91 y $170.36 \mathrm{~g}$ respectivamente, valores entre los cuales difieren significativamente $(\mathrm{P}<0.01)$, determinándose que el balanceado aporta con mayor cantidad de proteína, no así los pastos donde su aporte proteico fue prácticamente bajo. El requerimiento de proteína de los cuyes en la fase de crecimiento va desde al 13 - $18 \%$ (Ramírez, 2012).

Al utilizar desechos de tuza de maíz más alfalfa en un porcentaje mínimo se registró un consumo de proteína de $346 \mathrm{~g}$ de proteína, siendo superior al registrado en el presente trabajo, esto se debe a que la alfalfa aporta un alto porcentaje de proteína en la dieta de las especies pecuarias (Bonilla, J. Usca, J. Díaz, H. 2010). Debiendo señalarse que los pastos Axonopus scoparios, Pennisetum sp, Pennisetum pupureum, Tripsacum laxum que se suministró a los cuyes disponían de 3.88, 11, 3.41 y $6 \%$ respectivamente (Grupo editores, 2016)

El contenido de grasa aprovechada en los cuyes al utilizar balanceado, Tripsacum laxum, Pennisetum sp, Pernisetum purpureum y Axonopus scoparius fue de 80.13, 34.29, 51.87, 55.26 y 58.08 g respectivamente, valores entre los cuales difieren significativamente $(\mathrm{P}$ $<0.01$ ), determinándose que el balanceado aporta con mayor cantidad de grasa, no así los pastos el contenido de lípidos fue bajo. La cantidad de grasa que ingieren los cuyes en la fase de crecimiento es 84.6 g (Bonilla, S. Usca, J. Díaz, H. 2014), superior a los registrados en el presente estudio, excepto al balanceado el cual se ajusta a los determinados en la cita mencionada, esto se debe a que los pastos carecen de lípidos en su estructura. Además, a que el Axonupus scoparius posee apenas 1,29 de extracto etéreo, el pasto elefante $0.06 \%$ de grasa (Grupo Latino, 2016).

La fibra total aprovechada por los cuyes en la fase de crecimiento al disponer en su alimentación balanceado, Tripsacum laxum, Pennisetum sp, Pernisetum purpureum y Axonopus scoparius fue de 147.07, 226.33, 275.47, 290.89 y 272.96 g respectivamente, valores que difieren significativamente $(\mathrm{P}<0.01)$ entre si, determinándose que el balanceado aporta con menor cantidad de fibra, mientras que los pastos disponen de un alto contenido de fibra. Esto sucede debido a que el contenido de fibra en los forrajes depende del estado fisiológico del mismo al momento del corte, además la fibra del alimento es desdoblada por los microorganismos del ciego del tracto digestivo de los 
cuyes. El contenido de fibra de los pastos Axonopus scoparios, Pennisetum sp y Pennisetum pupureum que se suministró en los cuyes reportan valorse de: 27.79, 20, 6.20 y $6 \%$ respectivamente (Grupo editores, 2016).

La fibra detergente neutra (FDN) aprovechada de los pastos Tripsacum laxum, Pennisetum sp, Pernisetum purpureum y Axonopus scoparius fue de 724.43, 1071.53, 1146.59 y $1068.62 \mathrm{~g}$ respectivamente, valores entre los cuales difieren significativamente $(\mathrm{P}<0.01)$, determinándose que el Tripsacum laxum dispone de una menor cantidad de FDN, mientras que el Pernisetum purpureum dispone de la mayora cantidad. Esto sucede quizá se deba a factores fisiológicos y nutricionales de cada pasto.

En comparación al contenido de FDN entre la gramíneas y la leguminosas, el contenido de FDN en las leguminosas es menor que le contenido an las gramíneas, debido al alto grado de lignificación de las mismas en las leguminosas (Dupchak, 2003).

La fibra detergente ácida (FDA) aprovechada por los cuyes de los pastos Tripsacum laxum, Pennisetum sp, Pernisetum purpureum y Axonopus scoparius

fue de 484.21, 601.45, 636.44 y $660.14 \mathrm{~g}$ respectivamente, valores entre los cuales difieren significativamente $(\mathrm{P}<0.01)$, determinándose que el Tripsacum laxum dispone menor cantidad de FDA, mientras que el Axonopus scoparius reporta una disponibilidad es mayor. Esto se debe a factores intrínsecos propios de las especies forrajeras. Para la valoración de los mismos se utiliza la digestibilidad que es una medida biológica de la calidad de los alimentos y en ella intervienen factores dietarios como la composición química de los alimentos: La composición química de una especie forrajera puede ser diferente en función a las condiciones de manejo nutrición, el estado fenológico, siendo la fibra el nutriente que ejerce mayor influencia en la digestibilidad. Por lo tanto la digestibilidad de los contenidos celulares es mucho más alto que la digestibilidad de los componentes de la pared celular influenciados fuertemente por el grado de lignificación. Cañas (1995)

El bajo contenido de nutrientes esenciales como la proteína y su baja digestibilidad son considerados factores limitantes de las gramíneas tropicales, lo cual influye negativamente en el aprovechamiento adecuado en la producción animal. El consumo ligada a la calidad del forraje está asociada con el estado de fenológico, la especie y los factores del medio ambiente (Pirela, 2005).

A medida que la especie forrajera madura su estado fenológico disminuye la digestibilidad de la fibra Detergente Ácido (FDA), la cual es lo que queda después de una digestión de la pared celular con detergente ácido y abarca a la celulosa y la lignina, y tiene una correlación negativa con la digestibilidad del forraje. A mayor FDA, menor digestibilidad y menor contenido energético (Centeno, 2001). 
Tabla 1. Comportamiento productivo de los cuyes bajo el efecto de gramíneas tropicales

\begin{tabular}{llllllllllll}
\hline & \multicolumn{1}{l}{ Alimento de Cuyes } \\
\cline { 2 - 9 } Variables & Balanceado & Guatemala & Maralfalfa & Elefante & \multicolumn{2}{c}{ Gramalote } \\
\hline Peso Inicial (g) & 425.00 & a & 385.75 & a & 392.63 & a & 416.38 & a & 428.38 & a \\
Peso final (g) & 724.25 & a & 625.75 & a & 695.25 & a & 684.50 & a & 713.88 & a \\
Consumo MS (g) & 2230.63 & a & 2079.91 & a & 2266.68 & a & 2296.76 & a & 2274.11 & a \\
Heces producida (g) & 345.11 & a & 365.29 & a & 362.19 & a & 323.28 & a & 338.20 & a \\
MS aprovechada (g) & 1885.52 & a & 1714.62 & a & 1904.49 & a & 1973.47 & a & 1935.90 & a \\
Proteína aprovechada (g) & 305.45 & a & 133.40 & b & 151.17 & b & 149.91 & b & 170.36 & b \\
Grasa aprovechada (g) & 80.13 & a & 34.29 & b & 51.87 & b & 55.26 & b & 58.08 & b \\
Fibra aprovechada (g) & 147.07 & b & 226.33 & a & 275.47 & a & 290.89 & a & 272.96 & a \\
FDN aprovechada (g) & & & 724.43 & b & 1071.53 & a & 1146.59 & a & 1068.62 & a \\
FDA aprovechada (g) & & & 484.21 & a & 601.45 & a & 636.44 & a & 660.14 & a \\
\hline
\end{tabular}

Letras iguales no difieren significativamente según Tukey $(\mathrm{P}<0.05)$.

T0: Balanceado

T1: Tripsacum laxum (Pasto Guatemala)

T2: Pennisetum sp (Maralfalfa);

T3: Pennisetum purpureum (Pasto Elefante);

T4: Axonopus scoparius (Gramalote).

\section{Conclusiones.}

- Ala utilizar diferentes pastos tropicales frente al balanceado el peso final y consumo de materia seca no vario significativamente, aunque se puede demostrar mejor aprovechamiento al utilizar pasto gramalote se obtuvo un peso final de $713.88 \mathrm{~g}$ y al utilizar balanceado $724.25 \mathrm{~g}$, y un consumo de materia seca de 2274.11 y 2230.63 respectivamente.

- Las gramíneas forrajeras tropicales son escasas en proteína y grasa, aunque la superiores en fibra al contrastar con los pastos que se utilizó en la alimentación de los cuyes.

- La fibra detergente Neutra (FDN) y (FDA) ácida en el pasto Tripzacum laxum es inferior con relación al Pennisetum sp, pennisetum purpureum y Axonopus scoparius.

\section{Referencias Bibliográficas}

Acosta, A. Díaz, H. Trujillo, J. 2010. Evaluación de tres concentrados comerciales en la etapa de crecimiento-engorde de cuyes. Tesis de Grado. FCP - ESPOCH. Riobamba Ecuador. 
Argote Fe, Velasco R, Paz Pc. 2007. Estudio de métodos y tiempos para obtención de carne de Cuy (Cavia porcellus) empacada a vacío. Facultad de Ciencias Agropecuarias; 5 (2):103-111.

Bonilla, J. Díaz, H. Usca, J. (2010). Utilización de la cabuya agave americana como suplemento alimenticio para cuyes en las etapas de crecimiento - engorde y gestación - lactancia. Tesis de Grado. FCP - ESPOCH. Riobamba Ecuador.

Bonilla, S. Usca, J. Díaz, H. 2014. Utilización de diferentes niveles de maíz de desecho con tusa molida más melaza en la alimentación de cuyes. Tesis de Grado. Fcp Espoch. Riobamba Ecuador.

Casa, C. 2008. Efecto de la utilización del forraje verde hidropónico de avena, cebada, maíz y trigo en la alimentación de cuyes. Tesis de Grado. Facultad de Ciencias Pecuarias, Escuela Superior Politécnica de Chimborazo. Riobamba, Ecuador. pp $62-71$.

Chauca de Zl. 1997. Producción de cuyes (Cavia porcellus) Organización de las Naciones Unidas para la Agricultura y la Alimentación. Roma, Italia. http://www.fao.org/docrep/W6562S/W6562S00.htm

Coronado Sm. 2007. Manual técnico para la crianza de cuyes en el Valle del Mantaro. Talleres Gráficos PRESSCOM; Huancayo, Perú.

http://es.scribd.com/doc/58472339/2/Propiedades-y-Valor-Nutritivo-de-la-Carnede-Cuy.

Gómez, M. 2007. Evaluación del forraje verde hidropónico de maíz y cebada con diferentes dosis de siembra para las etapas de crecimiento y engorde de cuyes. Tesis de Grado. Facultad De Ciencias Pecuarias, ESPOCH. Riobamba, Ecuador. pp.62 71.

Grupo Latino, 2016. Pastos y Forrajes para ganado. Primera Edición. Colombia. 102 p.

Londo, V. Usca, J. Díaz, H. Y Trujillo, V. 2014. Utilización de diferentes niveles de zeolitas en el balanceado, para la alimentación de cuyes durante las etapas de crecimiento - engorde. Tesis de Grado. FCP - ESPOCH. Riobamba Ecuador.

Sánchez, A., Sánchez, S., Godoy, S., Díaz, R. Y Vega, N. 2009. Gramíneas tropicales en el engorde de cuyes mejorados sexados (Cavia porcellus Linnaeus) en la zona de la Maná. Facultad de Ciencias Pecuarias, Universidad Técnica Estatal de Quevedo. Mocache, Los Ríos, Ecuador. Publicado en Ciencia y Tecnología 2: pp. 25-28. 2009. Disponible en http://dialnet.unirioja.es/servlet/articulo?codigo $=4053241$

Sandoval, H. 2013. Evaluación de diferentes tipos de dietas en cobayos en crecimiento. Tesis de Grado. Carrera de Medicina Veterinaria y Zootecnia, Facultad de Ingeniería Agronómica. Universidad Técnica de Ambato. Cevallos, Ecuador. pp. 52 a 57.

Sayay, M. 2010. Utilización de dos variedades de maíz en la alimentación de cuyes en la etapa de crecimiento - engorde. Tesis de Grado. Facultad de Ciencias Pecuarias, Escuela Superior Politécnica de Chimborazo. Riobamba. Ecuador. pp. 45 - 75.

Solari G. 2010. Ficha Técnica de Crianza de cuyes. Soluciones Prácticas-ITDG. Lima, Perú.

http://www.solucionespracticas.org.pe/fichastecnicas/pdf/Crianza\%20de\%20cuyes. pdf 
Villa, S. Díaz, H. Usca, J. 2014. Niveles de afrecho de maíz en dietas para cuyes en las etapas de crecimiento - engorde y gestación - lactancia. Tesis de Grado. FCP Espoch. Riobamba Ecuador.

Xicohtencatl-Sánchez Pascual G, Barrera-Zúñiga Samuel, Tiodolo Orozco-Orozco, Torres-Sandoval Sigfredo Fidel Mar, Monsivais-Isiordia Roberto. 2013. Parámetros productivos de cuyes (Cavia porcellus) del nacimiento al sacrificio en Nayarit, México. Abanico Veterinario.

Cañas, R. 1995. Alimentación y Nutrición Animal. Colección en Agricultura Facultad de Agronomía Pontificia Universidad Católica de Chile.

Centeno, A. 2001. Silaje de alfalfa http://www.com/eforrajes/ver_articulo.asp?ver=7083.

Pirela, M. 2005. Valor nutritivo de los pastos tropicales. Instituto Nacional de $\begin{array}{llll}\text { Investigaciones } & \text { Agrícolas. } & \text { 2005, 176-177 }\end{array}$ URL:http://www.avpa.ula.ve/docuPDFs/libros_online/manualganadería/seccion3/ar ticulo6- s3.pdf

Dupchak, K. 2003. Ndf Digestibilities: a New Analysis to Evaluate Forage Quality. Manitoba Agriculture, Food and Rural Initiatives Nutrition Update. Vol. 14 (1). 
PARA CITAR EL ARTÍCULO INDEXADO.

Luis Rojas Oviedo, L. R. O., Noboa Abdo, T., Shagñay Rea, S., \& Luis Condo P. (2020). Alimentación de cuyes en la fase de crecimiento en base a gramíneas tropicales de Morona Santiago. ConcienciaDigital, 3(2.2), 50-59. https://doi.org/10.33262/concienciadigital.v3i2.2.1245

\section{Liencia}

El artículo que se publica es de exclusiva responsabilidad de los autores y no necesariamente reflejan el pensamiento de la Revista Conciencia Digital.

El artículo queda en propiedad de la revista y, por tanto, su publicación parcial y/o total en otro medio tiene que ser autorizado por el director de la Revista Conciencia

\section{Digital.}

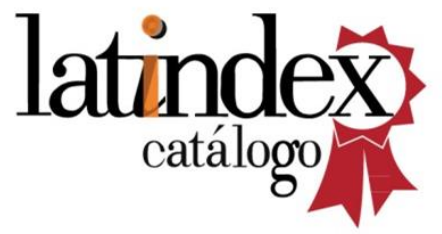

